\title{
THE ELLIP'TIC FUNC'IIONS.
}

The Applications of Elliptic Functions. By ALFRED GEORGE Greenhill, M.A., F.R.S., Professor of Mathematics in the Artillery College, Woolwich. London and New York, Macmillan \& Co., 1892. 8vo, pp. $x i+35 \%$.

IN recent years there has been a tendency to regard pure and applied mathematics as subjects to be kept clearly distinct. 'The increasing necessity for close specialization, occasioned by the enormous yearly ontput of memoir literature, is no doubt largely responsible for this unfortunate condition of things, but there are other elements which enter into the question. To a large class of pure mathematicians the lack of logical rigor in the current proofs of physical theorems is abhorrent; to them it seems that a treatise on any subject in pure mathematics should use methods peculiar to that subject, and that no appeal should be made to extraneous physical considerations. The applied mathematician, on the other hand, is too apt to speak scornfully of the works of his rival. It is indisputable that the problems which confront the physicist of to-day require for their adequate treatment the most refined methods in pure analysis. It is, we believe, equally certain that many portions of modern mathematics must remain, so to speak, in the air, until they are connected with the physical considerations from which they originated. Professor Greenhill's sympathies are largely with the practical man as against the theorist; the first words of his introduction are a quotation from Fourier in which occur the words "les questions vagues et les calculs sans issue." At the same time he has earned for himself a high reputation by his researches on the complex multiplication of elliptic functions, a subject withont any very immediate applications. Professor Greenhill has rendered an important service to the mathematical public by showing how easily elliptic functions lend themselves to the solution of dynamical and other problems; an especially valuable feature of his treatise is the free use which he has made of the Weierstrassian functions.

In the first chapter there is a detailed treatment of pendnlum motion, and in the course of this discussion several of the fundamental properties of $\operatorname{sn} u, \operatorname{cn} u, \operatorname{dn} u$ are established. It is shown that they have a real period and that they may degenerate into circular and hyperbolic functions. There are obvious advantages in beginning the subject with a concrete example; but we doubt whether this is the way " best calculated to define the elliptic functions, and to give the student an idea of their nature and importance." 'The all-important, 
property of an elliptic function is its double periodicity, but all discussion of this is postponed till page 254 .

In the second chapter there is an exhaustive account of the numerous forms which can be taken by elliptic integrals, and free use is made of the inverse notation $\mathrm{sn}^{-1}, \mathrm{cn}^{-1}, \mathrm{dn}^{-1}, \wp^{-1}$. In the next chapter there are many geometrical and mechanical illustrations of the elliptic functions. Without entering into details we shall show how some of these problems lead to elliptic integrals.

(1) 'The equation for a central orbit described under a force $P$ to the pole is

$$
P=h^{2} u^{2}\left(u+d^{2} u / d \theta^{2}\right) .
$$

Hence, when $P=\mu u^{3}+v^{\prime \prime}$,

$$
d \theta=d u / \sqrt{a+b u^{2}+c u^{4}} .
$$

(2) Let $A B$ be an elastic rod with two rigid arms $A a, B b$ at its extremities; and let two equal and opposite forces $T$ be applied at $a, b$ so as to act inwards. The rod being straight when unstrained, what is its form when bent? (See Minchin's "Statics," vol. II., p. 204).

Let $a, b$ lie in the axis of $x$. For the equilibrium of the portion $a A P$, where $P$ is a point $(x, y)$ of the rod, it is necessary that the moment of ' $T$ ' about $P$ be equal to $A / \rho$, where $A$ is the flexural rigidity and $\rho$ the radius of curvature. 'The resulting equation $T y=\rho$ leads to $A d^{2} \psi / d s^{2}=T d y / d s$ $=-T \sin \psi$; an equation of the same type as that which arises in pendulum motion.

(3) Captain Sumner's method determines the position of a ship at sea by the intersections of two small circles, known as circles of position. The projection of a circle of position upon a Mercator chart has for its equation

$$
\begin{array}{ll}
\text { either } & \cosh y / c=\sec \alpha \cos x / c \text {, } \\
\text { or } & \sinh y / c=\tan \beta \cos x / c \text {. }
\end{array}
$$

In either case $d s / d x$ leads to an elliptic integral.

(4) Let a surface of revolution be formed by the revolution of a plane curve round the axis $O x$, and let a geodesic on this surface be projected upon a plane perpendicular to this axis. Let $r, \theta$ be the polar coördinates of a point on this projection. If the surface is formed by the revolution of a catenary, we have the equations

$$
\begin{gathered}
r=a \cosh x / a, \\
r^{2} d \theta / d s=b, \\
d s^{2}=d x^{2}+r^{2} d \theta^{2}+d r^{2} .
\end{gathered}
$$


These lead to

$$
d \theta=b d r / \sqrt{\left(r^{2}-a^{2}\right)\left(r^{2}-b^{2}\right) .}
$$

In the fourth chapter the addition theorems are proved for Jacobi's functions, and there is an interesting account of the poristic theorems connected with Poncelet's polygons. Also many applications are given of elliptic functions to spherical trigonometry.

The subjects of the remaining chapters (v. to x.) are the algebraical form of the addition theorem; the elliptic integrals of the second and third kind; the elliptic integrals in general and their applications; the double periodicity of the elliptic functions; the resolution of the elliptic functions into factors and series; and the transformation of elliptic functions. In these chapters the chief properties are stated in Weierstrass's as well as in Jacobi's notation, and in connection with these properties there are many illustrations. For example, the chief formulæ are used, with much skill, in the discussion of the revolving chain, the tortuous elastica, the spherical pendulum and the top, and the trajectory of a projectile for the cubic law of resistance. Particular mention should be made of the interesting treatment of the motion of a rigid body about a fixed point, when acied on by no forces, and of the accompanying account of various geometric constructions for the motion, including Sylvester's method for measuring the time (see Routh's "Rigid Dynamics," vol. II.).

As a geometric illustration of the importance of Weierstrass's formulæ in $\wp$ - and $\sigma$-functions, we will show that some of the leading properties of the Cartesian can be derived from standard formulæ in Schwarz's " Formeln und Lehrsätze zum Gebrauche der elliptischen Functionen.”*

Let

$$
z=\int_{\infty}^{m} d w / \sqrt{4 w^{3}-g_{2} w-g_{3}}, \text { or } \quad w=\wp(z),
$$

where $g_{2}, g_{3}$ are real, and $g_{2}{ }^{3}-2 g^{2} g_{3}{ }^{2}$ is positive. Let the real roots $e_{1}, e_{2}, e_{3}$ of the equation $4 x^{3}-y_{2} x-g_{3}=0$ be in descending order of magnitude. The parallelogram of periods is a rectangle formed by a real period $2 \omega_{1}$ and by a purely imaginary period $2 \omega_{y}$. Let $0, a, \omega_{1}-a, \omega_{1}, \omega_{1}+a$, ${ }_{2} \omega_{1}-a, 2 \omega_{1}$ be points on the side $\left(0\right.$ to $\left.2 \omega_{1}\right)$, and let lines 1 ,

* The method employed in the text agrees essentially with that of Prof. Greenhill; the relations for the focal distances of a point are simplified by the use of the triple focus, and thus assume a more final form. The formulæ in the text have been published by Prof. Morley in the Haverford College Studies. 
$2,3,4$ be drawn through $a, \omega_{1}-a, \omega_{1}+a, 2 \omega_{1}-a$ parallel to the axis of imaginaries to terminate in the opposite side of the parallelogram of periods. When $z$ describes the line 1 we have, in circular coördinates,

$$
z+\bar{z}=2 a .
$$

In the plane of $\wp z$ (or shortly the $\wp$-plane) the circular coördinates are $\wp z, \wp \bar{z}$ (or shortly $\wp, \bar{\wp}$ ). Let $c$ denote $\wp 2 a$. By the addition theorem, as in Halphen, vol. II. p. 335,

$$
\left[8 \bar{\varnothing}+c(\wp+\bar{\varnothing})+g_{2} / 4\right]^{2}=4(\wp+\bar{\varphi}+c)\left(c \varnothing \bar{\varnothing}-g_{\mathrm{s}} / 4\right) \text {. }
$$

The equation is that of a Cartesian, since the highest power of $\wp$ is multiplied by $(\bar{\varnothing}-c)^{2}$. The triple focus has for its coördinates $c, c$, and corresponds to the point $z=2 a$. The single foci are at $e_{1}, e_{2}, e_{3}$. The curve depends on a single constant $c$ or $\wp 2 a$. It therefore includes the representations of four lines in the rectangle, viz. 1,2,3,4. For when $z$ is at any one of the points $a, \omega_{1}-a, \omega_{1}+a, 2 \omega_{1}-a$, the values of $\wp 2 z$ and $8 \% 2, a$ are the same. The lines 1 and 4 give the same values of $\wp z$ and give the outer oval of the Cartesian; while the lines 2 and 3 give the inner oval.

Let each point $8 \%$ be denoted (to periods près) by its parameter $z$, with the exception of the three points $e_{1}, e_{2}, e_{3}$. 'The point $e_{3}$ lies on the real axis outside the outer oval and $e_{2}, e_{1}$ lie within the inner oval. Starting from $e_{3}$, the real axis meets the curve in the points $\omega_{3}+a, \omega_{2}+a, \omega_{1}+a, a$, where $\omega_{2}=-\left(\omega_{1}+\omega_{3}\right)$. For the sake of symmetry let us denote a point on the curve by the parameter $a+z$, in preference to $z$; then for points on the outer oval $z$ is a pure imaginary, and for points on the inner oval $z-\omega_{1}$ is a pure imaginary (periods not counting). Since

$$
\left\{\varnothing\left(z+\omega_{\lambda}\right)-e_{\lambda}\right\}\left\{\wp z-e_{\lambda}\right\}=\left(e_{\mu}-e_{\lambda}\right)\left(e_{\nu}-e_{\lambda}\right),
$$

it follows that the point $a+z+\omega_{\lambda}$ is derived from the point $a+z$ by quasi-inversion (i.e., ordinary inversion plus reflexion in the real axis). Hence the point $a+z+\omega_{\lambda}$ is the inverse of the point $a-z$. The curve is therefore its own inverse with regard to any focus.

In formulæ (3), p. 47 of Schwarz's "Formeln und Iehrsätze," put

$$
a=0, \quad b=2 a, \quad c=a+z, \quad d=a-z .
$$


'Therefore

$$
\begin{aligned}
& a^{\prime}=2 a, \quad b^{\prime}=0, \quad c^{\prime}=z-a, \quad d^{\prime}=a+z, \\
& a^{\prime \prime}=a+z, \quad b^{\prime \prime}=a-z, \quad c^{\prime \prime}=0, \quad d^{\prime \prime}=-2 a .
\end{aligned}
$$

The formula (5) p. 48, namely,

$$
\begin{gathered}
\left(e_{\mu}-e_{\nu}\right) \sigma_{\lambda} a \sigma_{\lambda} b \sigma_{\lambda} c \sigma_{\lambda} d+\left(e_{\nu}-e_{\lambda}\right) \sigma_{\mu} a^{\prime} \sigma_{\mu} b^{\prime} \sigma_{\mu} c^{\prime} \sigma_{\mu} d^{\prime}, \\
+\left(e_{\lambda}-e_{\mu}\right) \sigma_{\nu} a^{\prime \prime} \sigma_{\nu} b^{\prime \prime} \sigma_{\nu} c^{\prime \prime} \sigma_{\nu} d^{\prime \prime}=0,
\end{gathered}
$$

becomes

But

$$
\Sigma\left(e_{\mu}-e_{\nu}\right) \sigma_{\lambda} 2 a \sigma_{\lambda}(a+z) \sigma_{\lambda}(a-z)=0 .
$$

$\frac{\sigma_{\lambda}(a+z) \sigma_{\lambda}(a-z)}{\sigma(a+z) \sigma(a-z)}=\sqrt{\xi \rho(a+z)-e_{\lambda} ;\left\lceil\rho(a-z)-e_{\lambda}\right\}}=\rho_{\lambda}$

where $\rho_{\lambda}$ is the focal distance; and, similarly,

$$
\frac{\sigma_{\lambda} \approx a}{\sigma 2 a}=\sqrt{\alpha_{\lambda}}
$$

where $\alpha_{\lambda}$ is the distance from the triple focus to a single focus. Hence Schwarz's formula gives, after division by $\sigma 2 a \sigma(a+z) \sigma(a-z)$,

or, since

$$
\Sigma\left(e_{\mu}-e_{v}\right) \sqrt{\alpha_{\lambda}} \rho_{\lambda}=0 ;
$$

$$
\begin{gathered}
e_{\mu}-e_{\nu}=-\left(\alpha_{\mu}-\alpha_{\nu}\right), \\
\Sigma\left(\alpha_{\mu}-\alpha_{v}\right) \sqrt{\alpha_{\lambda}} \rho_{\lambda}=0 .
\end{gathered}
$$

It can be proved, in a similar manner, that

$$
\begin{gathered}
\sqrt{\alpha_{2} \alpha_{3}} \rho_{1}-\alpha_{2} \alpha_{3}=\sqrt{\alpha_{3} \alpha_{1}} \rho_{2}-\alpha_{3} \alpha_{2}=\sqrt{\alpha_{1} \alpha_{2}} \rho_{3}-\alpha_{1} \alpha_{2} \\
=\frac{1}{2}\left(\rho^{2}-\alpha_{2} \alpha_{3}-\alpha_{3} \alpha_{1}-\alpha_{1} \alpha_{2}\right),
\end{gathered}
$$

where $\rho$ is the distance of the point from the triple focus.

When the discriminant $g_{2}{ }^{3}-2{ }^{2} g_{3}{ }^{2}$ is negative, the periods $2 \omega_{1}, 2 \omega_{3}$ are conjugate. Also if $e_{2}$ be the real root, $e_{1}$ and $e_{3}$ are conjugate. Let

$$
\begin{aligned}
& \left.\omega_{2}=\omega_{1}+\omega_{2}\right)_{3}, \\
& \omega_{2}^{\prime}=\omega_{1}-\omega_{3} .
\end{aligned}
$$

Then $\omega_{2}$ is on the real axis, $\omega_{2}{ }^{\prime}$ on the imaginary axis. The curve which corresponds in the $\wp$-plane to $z+z=2 a$ is still 
a Cartesian, but there is now only one oval. One real focus and two imaginary foci are collinear. The property of inversion now belongs only to the focus $e_{2}$, and of the relations between the focal distances the only one which is free from imaginaries is

$$
\rho^{2}=2 \sqrt{\alpha_{3} \alpha_{1}} \rho_{2}+\alpha_{1} \alpha_{2}+\alpha_{2} \alpha_{3}-\alpha_{1} \alpha_{3},
$$

$\rho, \rho_{2}, \alpha_{2}$ having their former meaning, and $\alpha_{1}, \alpha_{3}$ being the conjugate quantities $\wp 2 a-e_{1}$ and $\wp 2 a-e_{z}$.

The anthor states expressly in his preface that, as his book is addressed to trained mathematicians, he has considered it sufficient in many places to state the results without introducing the intermediate analysis. His opinion is that " by developing the analysis as it is required for some particular problem in hand, the student of applied mathematics will obtain a working knowledge of the subject of elliptic functions, such as he would probably never acquire from a study of a treatise like Jacobi's “Fundamenta Nova." This hand-to-mouth plan of taking up the subject has been carried, we think, too far. It would be a piece of pedantry to require that much space should be given to convergency considerations; but we are decidedly of opinion that such a proof as that on p. 202 ought not to have been insorted. It will certainly not bear close examination. It is unfortunate that so able a writer should show uniformly a contempt for processes and attach an undue importance to results; but much may be forgiven to one who can handle his symbols with such consummate ease. Wherever we open the book, we are struck with the freshness and originality of the treatment; though at times Mr. Greenhill's unconventionality is a trifle provoking. Was it necessary to raise the vexed question of pound-weight versus pound-mass on the first page, or to end the last chapter with the word, etc.?

We must call attention to one or two errors which have crept into the text. Chapter v. commences with the general symmetric quadri-quadric function of $x, y$. 'Two symmetric forms are to be distinguished:

(i.) $\alpha x^{2} y^{2}+2 \beta x y(x+y)+\gamma\left(x^{2}+4 x y+y^{2}\right)+2 \delta(x+y)+\epsilon=0$,

which states that $y$ is one of the polar pair of $x$ with regard to a fixed quartic

$$
\alpha t^{4}+4 \beta t^{3}+6 y t^{2}+4 \delta t+\epsilon=0,
$$

and

(ii.) the same expression $+4 \mu(x-y)^{2}=0$, 
which is the general symmetric quadri-quadric relation. The second form is that to which any quadri-quadric relation can be brought by writing $x=\left(l x^{\prime}+m\right) /\left(l^{\prime} x^{\prime}+m^{\prime}\right)$. This may perhaps be inferred from the fact that the relation (ii.) contains five effective constants, while the general relation has eight, the ratios $l / m^{\prime}, m / m^{\prime}, l^{\prime} / m^{\prime}$ accounting for the difference. (For a formal proof see Halphen's " Fonctions Elliptiques," vol. Ir. p. 338). Prof. Greenhill falls into two errors. He takes the special form (i.) as equivalent to

$$
(\sqrt{X}-\sqrt{Y})^{2} /(x-y)^{2}=a(x+y)^{2}+4 b(x+y)+C,
$$

where he should take the general form (ii.), and then, to compensate, he regards $\left(l x^{\prime}+m\right) /\left(l^{\prime} x^{\prime}+m^{\prime}\right)$ as a function with four effective constants. On page 313 the incorrect statement is made that the elliptic functions of the $n^{\text {th }}$ parts of the periods are called by Klein modular functions. A sentence on page 175 is so worded as to convey to the reader the impression that, in the most general case, abelian functions are the one-valued functions of $p$ arguments which are met with when we consider the integrals

$$
\int\left(1, x, x^{2}, \ldots, x^{p-1}\right) d x / \sqrt{X}
$$

where $X$ is a rational integral function of $x$ of the degree $2 p+2$. These are obviously mere oversights on the part of the author. We have pointed them out because they may cause trouble to the beginner.

We believe that the many sterling merits of this treatise will cause it to pass rapidly through a first edition. We hope that Prof. Greenhill will be encouraged, by the success of this book, to write one on the applications of antomorphic functions. A sentence in his recent address before the London Mathematical Society indicates that he is interested in this subject.

Bryn Mawr, March 9, 1893.

J. HARKNESS. 